\title{
Une cytokine aux frontières de l'inflammation et de l'immunologie : l'interleukine-6 (IL-6)
}

Bien que l'interleukine-6 (IL-6) soit (vraisemblablement pour peu de temps) avant-dernière dans la nomenclature officielle des interleukines (voir glossaire, p.339), une masse considérable de données est d'ores et déjà disponible à son sujet. En témoigne le fait que l'une des équipes qui avaient indépendamment cloné son gène [1-5] vient, seulement deux ans plus tard, de cloner celui de son récepteur [6]. Ces progrès rapides sont alimentés par la convergence des recherches que suscite la multiplicité des effets des cytokines (voir m/s, $n^{o} 10$, vol. 4, p. 651): l'utilisation de la protéine produite par génie génétique permet de lui décrire des effets inattendus; celle d'anticorps dirigés contre elle permet de démontrer que l'IL-6 est responsable de toute une série d'effets biologiques définis de longue date et en quête d'un support moléculaire.

C'est en 1986 qu'on a constaté l'identité des séquences des ADNc proposées par plusieurs équipes pour caractériser des protéines douées de propriétés différentes : interféron $\beta-2$, ainsi dénommé en raison de son homologie partielle avec l'interféron $\beta$ [4]; protéine de $26 \mathrm{kDa}$ inductible dans les fibroblastes [2]; facteur de différenciation des lymphocytes B de type 2 (BSF2) [3] ; facteur de croissance des hybridomes et des plasmocytomes $[1,5]$. Très vite, il est apparu que l'IL-6 était douée de propriétés pro-inflammatoires (elle est l'un des supports de l'activité hepatocyte stimulatory factor), que ses effets sur la lignée lymphoïde s'étendaient aux lymphocytes $T$, et qu'elle intervenait dans l'hématopoïèse.

Définition moléculaire de l'IL-6. La séquence protéique de l'IL-6 humaine, déduite de celle de l'ADNc, comporte 212 acides aminés, incluant un peptide signal de 28 acides aminés. La seule cytokine avec laquelle l'IL-6 présente une homologie significative est le facteur de croissance de la lignée granuleuse (G-CSF). L'organisation des gènes de ces deux cytokines présente également certaines analogies avec cinq exons de taille comparable, séparés par quatre introns. Le gène de l'IL6 humaine est situé sur le chromosome 7. Ses régions de contrôle possèdent des séquences homologues de celles du gène $c$-fos, ce qui est cohérent avec l'observation que ces deux gènes partagent certaines de leurs caractéristiques de régulation.

L'IL-6 naturelle est glycosylée et migre en électrophorèse avec des masses moléculaires apparentes multiples : $25 \mathrm{kDa}, 30 \mathrm{kDa}$ et parfois $45 \mathrm{kDa}$. La présence de cette dernière forme dépend de la source cellulaire, sans que l'on sache encore si cette constatation est liée à une hétérogénéité fonctionnelle. Dans le même ordre d'idées, certaines cellules (monocytes) produisent un ARN messager de $1,6 \mathrm{~kb}$, en plus du messager fibroblastique classique de $1,3 \mathrm{~kb}$.

L'IL-6 : une cytokine ubiquitaire. L'IL-6 est produite par de nombreux types cellulaires: monocytes (beaucoup plus que macrophages résidents), lymphocytes $T$ activés, fibroblastes, cellules endothéliales, kératinocytes, astrocytes, cellules microgliales, cellules mésangiales du glomérule, chondrocytes... Cette production est déclenchée par de multiples signaux: lipopolysaccharides bactériens (LPS), virus à ARN bicaténaire, HIV, poly-I:C, tumor necrosis factor (TNF), interleukine-l (l'IL1), plateled derived growth factor (PDGF), epidermal growth factor (EGF), prostaglandine E2, rayonnement UVB, etc. Ces signaux ont une certaine sélectivité selon le type cellulaire: le LPS est plus efficace sur la lignée monocytaire, alors que l'ILl est plus efficace sur la lignée fibroblastique... Ainsi, la production d'IL-6 survient-elle en réponse aux agressions les plus diverses: infection virale, traumatisme mécanique, thermique, lumineux, mettant en jeu les cellules présentes au site de cette agression (peau, endothélium vasculaire, tissu de soutien, cartilage...); infection bactérienne, mettant en jeu la première ligne des défenses non spécifiques (cellules de la lignée monocytaire) ; agression mettant en jeu une réponse immunitaire $T$ spécifique (lymphocytes $\mathrm{T}$ activés).

La production d'IL-6 : un phénomène en cascade. La multiplicité des cellules et des signaux responsables de la production d'IL-6 conduit à une amplification en cascade. Les

\begin{tabular}{|c|ccc|}
\hline \multicolumn{5}{|c|}{ Tableau I } \\
\multicolumn{4}{|c|}{$\begin{array}{c}\text { CELLULES PRODUCTRICES D'IL-1, } \\
\text { DE TNF ET D'IL-6 }\end{array}$} \\
\hline \multirow{3}{*}{ Cytokine } & Production par cellules \\
\cline { 2 - 4 } & monocytaires & T & ubiquitaires \\
IL-1 & + & - & + \\
TNF & + & + & + \\
IL-6 & + & + & + \\
\hline
\end{tabular}




\section{${ }^{*}$ GLOSSAIRE DES INTERLEUKINES HUMAINES}

IL-1 : facteur (15-17 kDa) pyrogène produit par des cellules de la lignée monocyte-macrophage et cellules ubiquitaires, capable d'induire le relarguage d'autres cytokines par des cellules $T$ activées et des fibroblastes, la prolifération de fibroblastes et cellules endothéliales, le relarguage de prostaglandine E2; c'est un cofacteur pour l'activation et la prolifération de cellules $B$. Gène cloné.

IL-2 : facteur (15 kDa) produit par des cellules $T$ activées capable d'induire la croissance de cellules $T$ activées, de thymocytes, la production de cytokines par des cellules $T$, une génération de cellules cytotoxiques, la prolifération et la différenciation terminale en cellules productrices d'anticorps de cellules $B$ activées. Gène cloné.

IL-3 : facteur produit par des clones de cellules $T$ activées ou des lymphocytes du sang périphérique stimulés par une lectine, capable d'induire la croissance de cellules souche multipotentes et la production d'histamine par le tissu hématopoïétique. Gène cloné.

IL-4: facteur (15-20 kDa) produit par des cellules $T$ activées capable d'induire la prolifération de cellules $T$ ainsi que de cellules $B$ activées,

interconnexions sont particulièrement claires avec deux autres cytokines qui présentent de nombreux points communs avec l'IL-6 : les IL1 et les TNF (Tableau I). Comme l'IL-6, les IL-1 $\alpha$ et $\beta$ sont produites par les cellules monocytaires et par des cellules ubiquitaires. Comme l'IL-6, les TNF sont produits par les cellules monocytaires (TNF- $\alpha$ ) et par les lymphocytes T activés (TNF- $\beta$ ). Il y a donc souvent production 'concomitante de ces médiateurs. Si l'IL-6 n'induit pas les autres cytokines, celles-ci potentialisent sa production. A titre d'exemple (figure 1), le LPS induit la production d'IL-6, d'IL-l et de TNF par les macrophages; IL-l et TNF vont stimuler la production d'IL-6 par les macrophages et les fibroblastes de voisinage.

$\mathrm{m} / \mathrm{s} n^{\circ} 5$ vol. 5 , mai 89 l'expression du marqueur CD23 (récepteur de faible affinité pour la portion $F C$ des immunoglobulines $E)$ sur des cellules $B$ initialement au repos, et une sécrétion préférentielle d'immunoglobulines $E$ (IgE) par les cellules B. Gène cloné à partir de son homologue murin.

IL-5: facteur (50-60 kDa) produit par des cellules $T$, capable d'induire la différenciation des éosinophiles, d'influencer la sécrétion d'IgM et peut-être d'IgA par des cellules $B$ activées. Gène cloné à partir de son homologue murin.

IL-6 : facteur (20-35 kDa) pyrogène produit par des monocytes, des cellules $T$ et des cellules ubiquitaires, capable d'induire la prolifération d'hybridomes et de plasmocytomes, la sécrétion d'immunoglobulines par des cellules $B$ normales ou transformées, la génération de lymphocytes cytotoxiques, la production de protéines hépatiques de la phase aigue de l'inflammation; l'IL-6 influence l'hématopoïèse. Gène cloné.

IL-7: facteur (20-28 kDa) capable d'induire la prolifération de cellules pré- $B$, pro- $B$, et de certaines cellules $T$. Gène cloné à partir de son homologue murin.

Les effets de l'IL-6 : paracrines, endocrines et peut-être autocrines. L'IL-6 est un facteur de différenciation pour la lignée lymphoïde $B$, mais aussi $T$, et pour les cellules hématopoïétiques : elle intervient alors par un mode d'action paracrine, comparable à celui de la plupart des cytokines. Elle joue un rôle dans la fièvre et le déclenchement de la production des protéines de l'inflammation, dans des situations où elle devient dosable dans les liquides biologiques: c'est donc également (tout comme les TNF et les IL-1) un facteur endocrine. L'IL-6 stimule la croissance des plasmocytomes, qui sont capables d'exprimer son récepteur et de transcrire son gène: elle pourrait intervenir comme un facteur de croissance autocrine pour certaines tumeurs.
L'IL-6, facteur de différenciation pour les lymphocytes B. Dans la production des anticorps, l'IL-6 agit sur le lymphocyte $B$ après que celui-ci a reçu le signal antigénique et une première aide de la part des lymphocytes T helper. Contrairement à d'autres interleukines, qui agissent à la fois sur la prolifération et la différenciation des lymphocytes B, l'IL-6 agit essentiellement sur la différenciation des lymphocytes B normaux, à la fois en augmentant l'expression du gène de la chaîne lourde des immunoglobulines et en favorisant la production d'ARN messagers correspondant à la forme sécrétée de cette chaîne lourde. Cet effet s'exerce sur l'ensemble des classes d'immunoglobulines et il peut être reproduit in vivo par injection à la souris d'IL-6 recombinante. Des souris transgéniques produisant de fortes quantités d'IL-6 développent une hypergammaglobulinémie, des plasmocytomes non malins et des lésions glomérulaires.

L'IL-6 stimule la génération des lymphocytes $T$ cytolytiques. La génération des lymphocytes $T$ cytolytiques à partir de la population $\mathrm{CD}^{+}$est augmentée par l'IL-6. D'une façon générale, l'IL-6, comme l'IL-1, potentialise l'effet de l'IL-2 en augmentant l'expression du récepteur de cette interleukine sur les lymphocytes T activés.

IL-6 et lignée B en pathologie. Le rôle possible de l'IL-6 dans une croissance autocrine des plasmocytomes a été mentionné ci-dessus. En outre, l'IL-6 pourrait jouer un rôle dans la pathologie liée au virus d'EpsteinBarr, puisqu'elle favorise l'établissement in vitro de lignées lymphoblastoïdes B induites par ce virus.

IL-6 et hématopoï̀se. Tout comme d'autres interleukines (IL-1, IL-4...), l'IL-6 agit en synergie avec les colony-stimulating factors (CSF). Elle induit la production de GMCSF par les fibroblastes et les cellules endothéliales de la molle osseuse. L'IL-6 agit directement sur les cellules hématopoïétiques à deux niveaux. Très précocement, elle potentialise l'effet de l'IL-3 sur la différenciation de la cellule souche. Tardivement, elle a des effets comparables à ceux du G-CSF sur la lignée 


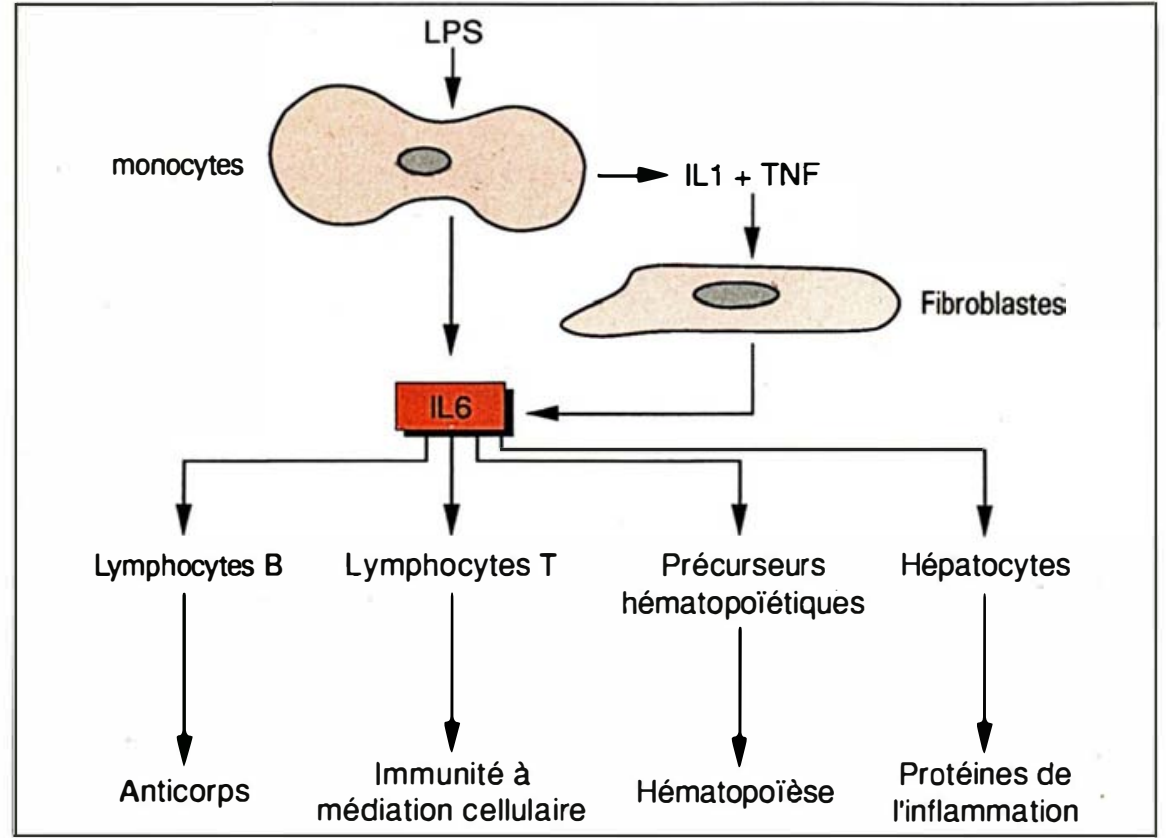

Figure 1. Production et effets de I'IL-6. L'IL-6 est produite par plusieurs types cellulaires, selon un processus comportant cascades et boucles d'amplification. Elle agit sur de multiples cibles, en synergie avec d'autres cytokines, différentes selon la cible.

granuleuse. L'IL-6 est capable d'induire la différenciation (avec arrêt de croissance) de lignées leucémiques myéloïdes, mais aussi de lignées tumorales non hématopoïétiques.

IL6 et inflammation. Si l'IL-6 est présente dans les épanchements articulaires inflammatoires, ses effets locaux sont moins bien définis que ceux de l'IL-l (l'IL-6 n'induit pas de production de prostaglandines). En revanche, au même titre que l'IL-1, l'IL-6 exerce un effet pro-inflammatoire général. Elle est pyrogène. Elle induit la production des protéines de l'inflammation par l'hépatocyte. Chacune deces deux cytokines induit la production d'un spectre limité de ces protéines et l'association des deux est en général synergique. D'une façon générale, cette synergie est potentialisée par les glucocorticoïdes. Cette synergie IL-1-IL-6- glucocorticoïdes pourrait être un phénomène plus général, puisqu'elle peut aussi être démontrée sur la cible lymphocytaire B [7]. En revanche, les glucocorticoïdes inhibent la production d'IL-6, aussi bien que celle d'IL-1. cible, impliqués dans la transmission intranucléaire du signal fourni par l'occupation du récepteur de l'IL-6. La place de l'IL-6. La multiplicité des cibles de la plupart des cytokines empêche de définir de façon absolue le rôle biologique propre de chacune d'entre elles. Il est clair que l'IL-6 joue un rôle au sein de plusieurs réseaux interconnectés. Un réseau d'activation lymphocytaire où elle participe - avec l'IL-2, l'IL-4, l'IL$1-$ aux réponses $B$ et $T$. Un réseau d'hématopoïèse où elle interagit, au même titre que d'autres interleukines, avec l'IL-3 et les CSF. Un réseau de réponse non spécifique aux agressions où elle est produite en conjonction avec et sous l'influence de l'IL-1 et du TNF. L'IL-6 pourrait y être le médiateur de certains des effets attribués à ces deux cytokines, caractérisées avant elle. Ainsi, ni les outils méthodologiques ni les hypothèses de travail ne manquent pour prédire une cascade de découvertes sur l'implication de ce médiateur dans les aspects les plus divers de la pathologie.

Pierre Galanaud Dominique Émilie Corinne Leprince Inserm U. 131 eucaryotes [6]. C'est une protéine de 468 acides aminés, dont la portion intracytoplasmique (82 acides aminés) n'a pas de domaine tyrosinekinase, et dont la portion extracellulaire se rattache à la superfamille des immunoglobulines. La portion extracellulaire peut être relarguée sous forme soluble et conserve alors une affinité pour le ligand. Il y a donc une possibilité d'interaction IL-6-récepteur dans le milieu extracellulaire, sans que la signification biologique de ce phénomène soit claire. L'IL-6 peut influencer l'expression de nombreux gènes parfaitement caractérisés (multiples protéines de l'inflammation, immunoglobulines). Des recherches, fondées sur l'analyse des régions régulatrices de ces gènes (définition des séquences impliquées dans la régulation de l'expression, recherche de protéines liant l'ADN dans ces régions...) devraient permettre de définir les facteurs, généraux ou spécifiques de

Mécanismes d'action de l'IL-6. Le (n)

(1)

\section{RÉFÉRENCES}

1. Billiau A. BSF2 is not just a differentiation factor. Nature $1986 ; 324: 415$.

2. Haegeman G, Content J, Volckaert G, et al. Structural analysis of the sequence coding for an inducible $26-\mathrm{kDa}$ protein in human fibroblasts. Eur J Biochem 1986; 625-32.

3. Hirano $\mathrm{T}$, Yasukawa $\mathrm{K}$, Harada $\mathrm{H}$, et al. Complementary DNA for a novel human interleukin (BSF-2) that induces B lymphocytes to produce immunoglobulin. Nature 1986 ; 324: 73-6.

4. Zilberstein A, Ruggieri R, Korn JH, et al. Structure and expression of cDNA and genes for human interferon-beta-2, a distinct species inducible by growth-stimulatory cytokines. EMBO J 1986 ; 5 : 2529-37.

5. Brakenhoff JPJ, De Groot ER, Evers RF et al. Molecular cloning and expression of hybridoma growth factor in Escherichia coli. J. Immunol. 1987 ; 139: 4116-21.

6. Yamasaki K, Taga Y, Hirata Y, et al. Cloning and expression of the human interleukin-6 (BSF-2/IFN-beta-2) receptor. Science 1988 ; 241 : 825-8.

7. Emilie D, Crevon MC, Auffredou MT, et al. Glucocorticosteroid-dependent synergy between IL-1 and IL-6 for human B lymphocyte differentiation. Eur J Immunol 1988; 18 : 2043-8. 